\title{
Formulación de políticas públicas en salud durante la pandemia de covid-19 en países americanos
}

\author{
Erick Landeros-Olvera ${ }^{1 *}$; Francisco Javier Arroyo-Cruz ${ }^{2}$; Mariela de Jesús Rodríguez-Hernández ${ }^{3}$
}

\begin{abstract}
RESUMEN
Introducción: La pandemia ha provocado una situación de respuesta inmediata de acciones globales no probadas y no evaluadas, se ha tenido poco tiempo para verificar resultados. Es imprescindible formular políticas públicas para establecer la normativa de dichas acciones en los programas en salud. Objetivo: Presentar una propuesta para la operacionalización de políticas de salud que sean encaminados a resolver problemas derivados de la pandemia por Covid-19. Desarrollo: Ante las epidemias registradas en la historia, las políticas de salud desarrollaron programas que ofrecieron soluciones de prevención y tratamiento. Ahora, la situación de emergencia ha obligado a trabajar para remediar, comprender y mejorar las políticas sanitarias en los países americanos. La Organización Mundial de la Salud ha hecho esfuerzos por contribuir a delimitar la pandemia a través de los distintos gobiernos de América, pero ¿Cómo se pueden formular políticas factibles si la situación en cada país ha presentado distintas estategias de contingencia sanitaria? La propuesta para la Formulación y ejecución de las políticas públicas de salud es la siguiente: 1.Definir el problema, 2.Generar soluciones, 3.Objetivo principal, 4.Redactar estrategias, 5. Operacionalización, 6.Planeación, 7.Ejecución, 8.Resultados, 9.Evaluación, 10.Retroalimentación. Conclusiones: Las políticas públicas de salud deben ser "adaptadas" a la realidad financiera y cultural de cada país, considerar retos actuales de salud; sin embargo, los profesionales de la salud, al proponer políticas públicas, deben estar conscientes de buscar respaldo del congreso, que sea la voz para representar la propuesta de quienes están en el lugar de los hechos.
\end{abstract}

Palabras clave: Políticas; Políticas públicas; Enfermería (DeCS).

1 Doctor en Ciencias de Enfermería. Benemérita Universidad Autónoma de Puebla. Facultad de Enfermería. Av. 25 Pte. 1304, Los Volcanes, 72410 Puebla, Pue. Tel: 2295500 ext. 5618.

ORCID: https://orcid.org/0000-0001-6270-1759

2 Estudiante de posgrado. Benemérita Universidad Autónoma de Puebla. Facultad de Enfermería. Maestría en Enfermería. Av. 25 Pte. 1304, Los Volcanes, 72410 Puebla, Pue. Tel: 2295500 ext. 5618. ORCID: https://orcid.org/00000003-2044-1412

3 Estudiante de posgrado. Benemérita Universidad Autónoma de Puebla. Facultad de Enfermería. Maestría en Enfermería. Av. 25 Pte. 1304, Los Volcanes, 72410 Puebla, Pue. Tel: 2295500 ext. 5618. ORCID: https://orcid.org/00000002-7960-280X

*Autor para correspondencia: franciscoarroyoc2004@gmail.com 


\section{INTRODUCCIÓN}

La pandemia producida por el nuevo coronavirus SARS-Cov-2 ha colocado al mundo en una situación de respuesta inmediata, gran parte de las intervenciones se realizaron $\sin$ la infraestructura adecuada y sin evidencia previa que pudieran sustentar las acciones globales en el sistema de salud de todos los países, que por ser un evento sin precedentes y por el poco tiempo que se ha tenido, se han mostrado resultados de investigaciones no evaluadas, tal como se presenta en la mayoría de los artículos que se han publicados durante la pandemia como "Preprints"1, 2. El impacto se ha reflejado en la incidencia de casos reportados a nivel mundial, hasta el 6 de enero de 2021 se han registrado $85,091,012$ casos confirmados $(585,557$ casos nuevos) y 1,861,005 defunciones (11,632 nuevas defunciones), con una letalidad global de $2.2 \%$; en América los casos reportados hasta el momento son 242,785 dando un porcentaje de distribución de $41.5 \%{ }^{3}$. Tanto la evidencia científica como los datos epidemiológicos, son elementos básicos para guiar a las políticas públicas de salud de cualquier país, las cuales tienen como principal función guiar las acciones para dar una respuesta socialmente organizada, a través del uso adecuado de los recursos humanos y materiales ${ }^{4}$. Estas políticas públicas tienen su operacionalización a través de los programas que de ellas emanen, es decir, llegan a concretarse cuando se transforman en servicios de acuerdo con las necesidades de salud de las comunidades ${ }^{5}$.

En el continente americano existen problemas de salud que requieren atención globalizada, por ejemplo, los esfuerzos que buscan establecer políticas públicas de salud que aborden problemáticas similares. Tal es el caso de la transición epidemiológica hacia las enfermedades crónicas que han marcado las principales causas de muerte y enfermedad 6 . A pesar de ser un problema de salud pública en común, en cada país, se presentan diferentes contextos y recursos para lograr concretar los programas derivados, tanto económicos como de servicios de salud, tal cual se ha presentado en la Organización para la Cooperación y el Desarrollo Económico (OCDE, ${ }_{2020}{ }^{78}$. Para que una política pública de salud llegue a reflejarse en los programas de salud de los países, debe realizarse un análisis previo de la situación y su formulación debe ser fundamentada en los resultados que arrojen las investigaciones de salud pública en conjunto con los resultados clínicos, para lograr un servicio eficiente, efectivo y más apegado al contexto real. En otras palabras, no es viable proponer políticas públicas de salud sin evidencia científica que sea parte del desarrollo social de la comunidad internacional ${ }^{9}$. Pero, ante la emergencia provocada por la pandemia de Covid-19 ¿Cómo se pueden formular políticas factibles? si la situación en cada país ha presentado distintas estrategias de contingencia sanitaria ${ }^{7}$.

El propósito de este ensayo es presentar una propuesta para la operacionalización de políticas públicas de salud que sean encaminados a resolver problemas derivados de la pandemia por Covid-19.

\section{PROBLEMAS COMUNES ¿SOLUCIONES COMUNES?}

A principios del siglo $X X$ en el continente americano, gran parte de las muertes fueron causadas por epidemias derivadas de la difteria, tosferina, tétanos, sarampión, tuberculosis, poliomielitis, el promedio de vida no sobrepasaba los 50 años $^{10}$. Ante ese problema, las políticas públicas de salud desarrollaron programas que ofrecieron cobertura a través de la vacunación universal, un programa derivado del análisis epidemiológico que, desde el punto de vista técnico, validó la propuesta política para lograr el máximo beneficio social posible. Sin embargo, a 
través del tiempo se ha identificado fallas en las políticas públicas de salud por la misma falta de evidencia científica ${ }^{11}$. Por un lado, en algunos sectores se ha presentado un rezago epidemiológico derivado de la pobreza y marginación de ciertas comunidades ${ }^{12}$, es decir, los pobres se siguen muriendo por las mismas enfermedades "aparentemente controladas" en pleno siglo XXI.

Por otro lado, la vacunación ha favorecido el aumento en el promedio de vida, pero como consecuencia, la aparición de enfermedades crónicas como la diabetes, enfermedades de corazón y la obesidad, que ahora aparecen cada vez en edades más jóvenes por la falta de políticas públicas de salud para enfrentar estilos de vida no saludables como el sedentarismo y la alimentación de comida chatarra que gestionaron un medio ambiente obesogénico ${ }^{13}$. En el continente americano, estas afecciones causan un gran impacto económico y social y han representado un reto de salud pública constante, y actualmente un factor de riesgo de mortalidad durante la pandemia causada por Covid 19, creando una crisis en materia de salud pública, económica y política ${ }^{14}$.

Esta situación de emergencia, ha obligado a trabajar para remediar, comprender y mejorar las políticas públicas de salud en los países americanos, la Organización Mundial de la Salud (OMS), ha realizado enormes esfuerzos por contribuir a delimitar la pandemia a través de los distintos gobiernos de América, diseñando recomendaciones de salud en conjunto con los representantes de todos los países americanos; sin embargo, los resultados no representan una norma, las políticas públicas de salud de la OMS son opcionales para el sistema de salud de todos los países y la razón es sencilla, pese a que la pandemia representa un problema de salud común, las soluciones no han sido necesariamente las mismas ${ }^{15}$. En cada país existen diferencias en el medio ambiente, la administración pública, los servicios sanitarios, la educación de la población y su diversidad cultural, esto significa que las acciones en las estrategias deben de adecuarse al contexto en que se esté desarrollando, considerando los recursos con los que cuenta cada país, ya que un país desarrollado es totalmente distinto en comparación con uno en vías de desarrollo ${ }^{16}$.

\section{FORMULACIÓN Y EJECUCIÓN DE LAS POLÍTICAS PÚBLICAS DE SALUD}

Lo anterior sin duda es determinante, pero en particular ante la emergencia sanitaria, cada país tomó sus propias decisiones para tomar acciones estratégicas. Algunos países americanos dieron prioridad a intereses políticos y económicos, otros antepusieron la opinión técnica de científicos y consideraron la experiencia vicaria que brindó China y países europeos a través del comportamiento de sus curvas epidemiológicas y la misma historia, cuando se presentaron epidemias en otras épocas ${ }^{17}$.

Pero independientemente de la estrategia, a casi un año de aparecer el nuevo coronavirus, se continúa evaluando las acciones con base a resultados, errores y aciertos, independientemente de los resultados económicos y la deuda que se generó en cada país, la evaluación principal es desde el punto de vista epidemiológico. Se han identificado diversas formas de interpretación de las curvas epidemiológicas, guiadas por la base de datos generada de cada país, en donde se indica el número de muertes acumuladas, el repositorio de datos de la Universidad Johns Hopkins ${ }^{18}$, permite construir las curvas respectivas con los datos que se consideren. Por ejemplo, con una tasa por cada 10,000 habitantes, situando el inicio de la curva a partir de la muerte número 100 como estrategia de covarianza, se puede observar que la curva tiene diferentes comportamientos en los países americanos hasta el mes de agosto del 2020, situación que expone una de las razones en la diversidad de acciones que cada país ha implementado ante la contingencia sanitaria (Figura 1). 
Figura 1. Variabilidad de las curvas epidemiológicas en países americanos

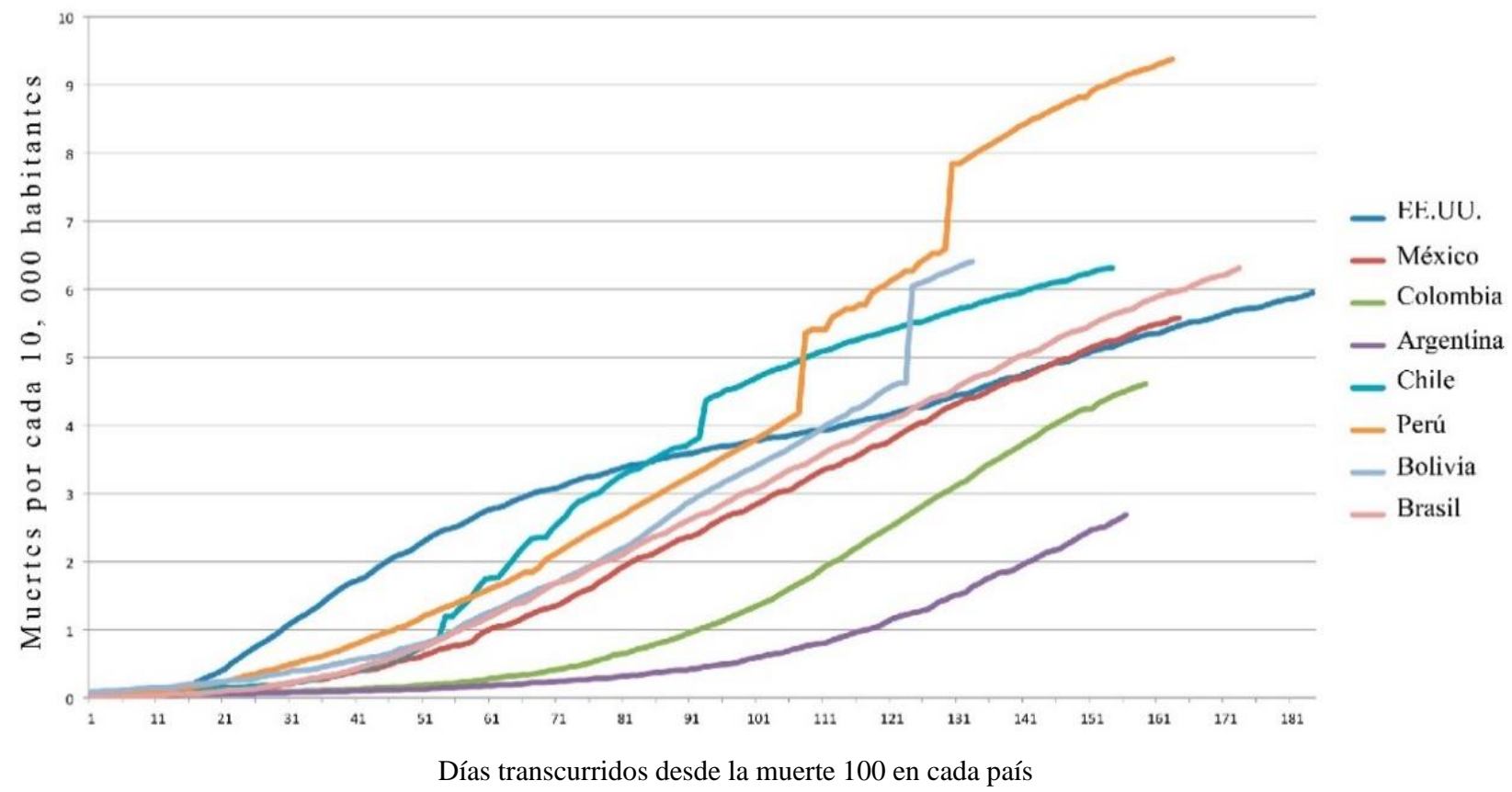

Fuente: Repositorio de datos COVID-19 del Centro de Ciencia e Ingeniería de Sistemas (CSSE) de la Universidad Johns Hopkins ${ }^{12}$

En algún momento todas las acciones que hayan tenido éxito deben de configurarse en políticas públicas de salud en esta nueva normalidad, en donde se puedan plantear el conjunto de objetivos, decisiones y estrategias para solucionar los problemas prioritarios que derivaron de la pandemia. Aunque es conocido que los intereses partidistas se dividen en la formulación y aprobación de políticas públicas ${ }^{19}$, se debe de colocar siempre la evidencia científica y la técnica de procedimientos por el bien común.

Para contribuir con este momento histórico, en este ensayo se propone 10 etapas para la formulación de una política pública de salud ajustada al contexto latinoamericano, adaptado del marco referencial de Mason $D$, et al. ${ }^{20}$. Primero, describir el problema de salud derivado de la pandemia, el cual debe ser definido, determinado bajo previa investigación estadística, epidemiológica, datos derivados de recursos humanos o materiales, ya que es necesario recopilar toda la información posible de manera fidedigna para justificar la política pública deseada. Segundo, generar alternativas de solución, en donde se proyecten las probabilidades, es decir, qué es posible y qué no con base a las siguientes preguntas: ¿qué implica?, ¿cuándo realizarla?, ¿dónde establecerla?, ¿por qué aplicarla? y ¿con qué recursos financieros se cuenta? Para identificar la factibilidad de una política pública de salud, se debe considerar como punto de partida que en la construcción de la agenda pública existe una compleja interconexión entre los problemas socio-sanitarios aparentes y reales, por lo cual se requiere considerar todos los escenarios posibles, 
ya que el problema planteado tiene que ser tratado como un fenómeno de salud con múltiples soluciones viables. Tercero, construir un objetivo principal, que contenga las alternativas de solución y que esté redactado de tal forma que abarque las respuestas a las preguntas anteriores. Se debe de recordar que, en este momento, existen demasiadas "opiniones" sobre lo que se debe 0 no ejecutar, porque la emergencia sanitaria de esta magnitud es un fenómeno con el cual no se había lidiado, por lo tanto, cada alternativa de solución debe derivar de evidencia científica y técnica publicada, misma que haya sido validada ${ }^{5}$. Cuarto, redactar las estrategias para cubrir el objetivo principal, considerando las acciones que van a operar a través de un programa, el cual tendrá que indicar las instancias que serán responsables de operarlo, identificar la comisión, el comité, la secretaría o el grupo representativo cuyas funciones impliquen directamente la responsabilidad de la ejecución. Quinto, el programa debe contener objetivos específicos para dar marcha a la operacionalización y estos se darán a conocer en los proyectos derivados de cada programa de acuerdo con el problema identificado, para resolver o prevenir. Sexto, cada proyecto del programa debe de mostrar sus productos esperados, es decir, con base a una planeación temporal, se debe de redactar las metas específicas y la fecha para obtener los resultados deseados. Cuando estos seis pasos estén establecidos, la política pública de salud puede ser sancionada por las autoridades correspondientes y se tiene la oportunidad de perfeccionarla, por esta razón, este paso tiene interrelación con todo el proceso, porque cada instancia de evaluación puede sugerir o solicitar cambios para ser aprobada. El tema de la evaluación de las políticas públicas de salud ha tomado auge progresivamente, en las últimas décadas como respuesta a la demanda ciudadana para una gestión eficaz, eficiente y transparente de los recursos públicos, algunas propuestas de políticas públicas de salud son formuladas por organizaciones como $\mathrm{OMS}^{21}$ a nivel internacional y otras al interior de cada país ${ }^{22}$ en el sistema interamericano. Por describir un ejemplo de la etapa de sanción, en México el Consejo Nacional de Evaluación de la Política de Desarrollo Social (Coneval) $^{23}$ es una instancia gubernamental mexicana con autonomía y capacidad técnica para generar información objetiva sobre la situación de la política social y la medición de la pobreza en el país, sus atribuciones le permiten mejorar la toma de decisiones en la sanción de políticas públicas de salud. Pero el Coneval no es la única instancia que debe de considerarse, para que una política pública sea aceptada, tiene que adaptarse a lo estipulado en el plan de desarrollo nacional, estatal o municipal, según sea el caso y la secretaría encargada es Planeación y Finanzas ${ }^{24}$ ya que es la instancia que promueve el proceso de planeación, recurso presupuestario, ejercicio responsable y congruente a las políticas públicas de salud que impulsen el bienestar como lo establece el Art $25^{\circ}$ que habla sobre el desarrollo del país ${ }^{25}$. En México, la facultad de proponer leyes la comparten en igualdad de circunstancias al ejecutivo, los integrantes del Congreso de la Unión y las Legislaturas Estatales, cabe mencionar que la Auditoría Superior de la Federación (ASF) ${ }^{26}$ posee el carácter de órgano técnico de fiscalización de la Cámara de Diputados, que tiene a su cargo la revisión del gasto público ejercido por el gobierno federal mexicano, los tres poderes de la unión, estados y municipios.

A nivel particular, por ejemplo, Tlaxcala es el único Estado de México que cuenta con una Secretaría de Políticas Públicas y Participación Ciudadana, encargada de diseñar, sugerir y ejecutar acciones de gobierno que generen interés público. Sin embargo, no siempre es consultada ya que la mayoría de las veces las políticas públicas de salud son decretadas por el poder ejecutivo y legislativo ${ }^{27}$. Continuando en el proceso, después de que la política pública haya sido sancionada y aceptada, el séptimo paso es la ejecución del programa, lo que prosigue, debe estar basada en el tiempo en que la política pública será aplicada o permitida, puede ser permanente o flexible al cambio o las situaciones con las que se presenten 
o comporte la epidemia o los casos. Esta fase está relacionada con el presupuesto asignado, ya que sin dinero no hay programas operables, luego entonces se debe de escribir con claridad, para que sea atractiva y considerada. Para lograrlo, independientemente de la temática propuesta (contratación de especialistas, recursos de protección, estrategias de acción en diferentes niveles de atención, continuidad de cuidados, salud mental, reglamento de sanidad en comercios y centros escolares, etc.) la ejecución del programa debe de estar justificada al margen de la prevención de la infección por Covid-1915. Octavo, obtener los productos finales y confrontar los resultados contra los indicadores que se tengan establecidos. Si la política fue bien planeada y sometida a un análisis de probabilidades, este punto debe coincidir con las predicciones de resultados que emanaron de las alternativas de solución del segundo punto. Noveno, la evaluación, -que a pesar de estar casi al final del proceso-, estará siempre presente en todas las fases del proceso en general. Evaluar cada una de las fases de la política pública de salud, da la oportunidad de corregir, implementar y redireccionar las estrategias para lograr el éxito de servir a la sociedad. Por último, el décimo paso, se enfocará a la retroalimentación y reestructura, paso que mejora el desarrollo de la política pública de salud con el paso del tiempo, por lo tanto, es una fase en continuo movimiento y relación con todas las demás fases, lo que resulte, siempre expone a la política pública a la transformación, para lograr su consolidación y tenga la posibilidad de ser replicada o considerada por otras entidades con diferentes gobiernos. La retroalimentación de una política pública de salud está expuesta a identificar barreras, exponer beneficios y plantear la factibilidad de réplica en diferentes zonas geográficas para lograr la universalidad.
Las políticas públicas de salud deben de ser "adaptadas" a la realidad financiera y cultural, considerando retos actuales de salud, no solo por la presencia del nuevo coronavirus, también por perfil epidemiológico específico de cada país y el medio ambiente que afecta los estilos de vida. Sin embargo, en la actualidad los profesionales de la salud como enfermeras y médicos, al proponer políticas públicas de salud deben estar conscientes que debe existir el respaldo de alguien en el congreso, que sea la voz para representar la propuesta, de lo contrario no sería posible, pese a que sea factible y con base a la evidencia. Para finalizar, la propuesta presentada en este ensayo para la formulación y ejecución de políticas públicas de salud, a diferencia del marco referencial de Mason D, et.al. ${ }^{16}$, considera características del medio político latinoamericano para contextualizarlo a las instituciones gubernamentales como mejor se considere, es flexible y puede servir de referente para que profesionales de la salud que no son actores políticos, puedan realizar propuestas en el congreso, ya que en ellos recae gran parte de la responsabilidad y cuidado de los pacientes de covid-19, su conocimiento y experiencia fundamentada en la ciencia y la técnica, realza la fortaleza para proponer políticas con base a las necesidades reales.

\section{CONFLICTO DE INTERESES}

Los autores declaran no tener ningún tipo de conflicto de intereses en relación con el ensayo.

\section{FINANCIAMIENTO}

Los autores declaran no haber recibido ningún tipo de financiamiento para la realización de este escrito.

\section{CONCLUSIONES}

\section{AGRADECIMIENTOS}


Nuestro agradecimiento a las personas que colaboraron con el logro de este ensayo. Georgina Mayela Núñez Rocha, José Antonio
Morales Santiago, Andrea Romero González y José Eusebio Arroyo Cruz.

Figura 2. Formulación y ejecución de las políticas públicas de salud

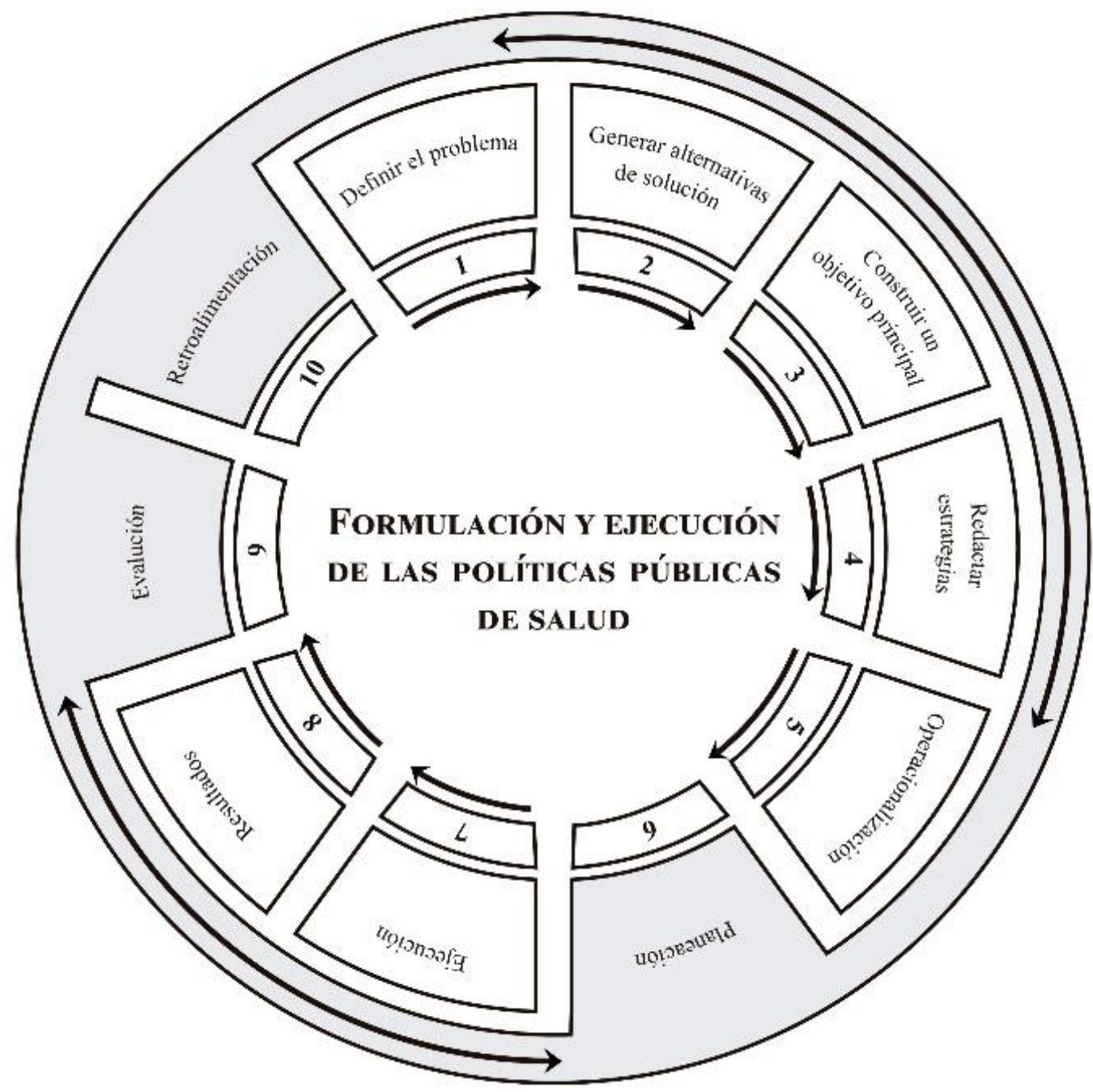

Fuente. Propia. Adaptado del marco referencial de Mason D, Gardner D, Hopkins F, O'GradyE. Policy \& Politics in Nursing and Health Care $^{20}$.

1. Fraser N, Brierley L, Dey G, Polka JK, Pálfy $M$, Coates JA. Preprinting a pandemic: the role of preprints in the COVID-19 pandemic. BioRxiv preprint doi: 
https://doi.org/10.1101/2020.05.22.1112

94.

2. Bourne PE, Polka JK, Vale RD, Kiley R. Ten simple rules to consider regarding preprint submission. PLoS Comput Biol 13(5): e1005473.

3. Panel de control de la enfermedad por coronavirus de la OMS (COVID-19). Ginebra:

Organización Mundial de la Salud, 2020. [Internet]. 2021 [citado 06 enero 2021] Disponible en: https://covid19.who.int/

4. Araneta F, Rosa M. La problematización de la realidad desde la mirada comunicacional para el diseño y gestión de políticas públicas. Actas de Periodismo y Comunicación [Internet]. 2018 [citado 22 septiembre 2020];4(2). Disponible en: https://www.perio.unlp.edu.ar/ojs/index. php/actas/article/view/5827

5. Barboza-Palomino, Caycho T, CastillaCabello H. Políticas públicas en salud basadas en la evidencia. Discusión en el contexto peruano. Salud pública de México [Internet]. 2017 [citado 19 septiembre 2020];59(1):2-3. Disponible en: http://dx.doi.org/10.21149/7881

6. Faus-Bertomeu A, Gómez-Redondo R. La reconstrucción de las causas de muerte por el método de MODICOD en el análisis demográfico-sanitario. De la CIE-9 a la CIE-10 a un nivel de cuarto dígito. EMPIRIA: Revista de Metodología de Ciencias Sociales [Internet]. 2018 May [cited 2020 Sep 19];40:167-95. Available from:

https://doi.org/10.5944/empiria.40.2018. 22015

7. Clavellina Miller JL, Domínguez Rivas MI. Implicaciones económicas de la pandemia por COVID-19 y opciones de política. 2020 [cited 2021 Jan 6]; Available from:

http://bibliodigitalibd.senado.gob.mx/ha ndle/123456789/4829
8. Rodríguez TG, de la Luz Pérez Reveles M, Bolívar HR. Efectos de las medidas de prevención y mitigación de la pandemia de COVID-19 sobre los índices de confianza en países de la OCDE. Contad adm. 2020;65(4):214.

9. Chaverri Chaves P, Arguedas Ramírez A. Políticas Públicas Basadas en Evidencia: una revisión del concepto y sus características. ABRA [Internet]. 2020 [citado 20 septiembre 2020];40(60):4976. Disponible en: https://doi.org/10.15359/abra.40-60.2

10. Orive N. Mortalidad infantil, malformaciones congénitas y transición epidemiológica: un reto para el sistema de salud. Revista Electrónica Dr. Zoilo E. Marinello Vidaurreta [Internet]. 2017 [citado 20 septiembre 2020];42(2). Disponible en: http://revzoilomarinello.sld.cu/index.php /zmv/article/view/1047/pdf_391

11. Chaverri Chaves $P$, Arguedas Ramírez A. Políticas Públicas Basadas en Evidencia: una revisión del concepto y sus características. ABRA [Internet]. 2020 [citado 20 septiembre 2020];40(60):4976. Disponible en: https://doi.org/10.15359/abra.4060.2

12. Brett $\mathrm{T}$, Ajelli $\mathrm{M}$, Liu Q-H, Krauland MG, Grefenstette JJ, van Panhuis, WG, Vespignani A, Drake JM, Rohani P. Detectar desaceleraciones críticas en sistemas epidemiológicos de alta dimensión. Plos Computational Biology. [Internet]. 2020 [citado 20 septiembre 2020]; 16(3): 1-19. Disponible en: https://doi.org/10.1371/journal.pcbi.100 7679

13. Kaczynski AT, Eberth JM, Stowe EW, Wende $M E$, Liese $A D$, McLain $A C$, Breneman $C B$, Josey MJ. Development of a national childhood obesogenic environment index in the United States: differences by region and rurality. 
International Journal of Behavioral Nutrition \& Physical Activity [Internet]. 2020 Jul 2 [cited 2020 Sep 19];17(1):1-11. Available from: https://ijbnpa.biomedcentral.com/article s/10.1186/s12966-020-00984-x

14. Webber R. COVID-19 and race: Protecting data or saving lives? International Journal of Market Research [Internet]. 2020 Sep [cited 2020 Sep 19];62(5):528-37. Available from: https://journals.sagepub.com/doi/full/10. 1177/1470785320946589

15. La OMS publica directrices para ayudar a los países a mantener los servicios sanitarios esenciales durante la pandemia de COVID-19 [Internet]. Organización Mundial de la Salud. 2020 [citado 14 septiembre 2020]. Disponible en: https://www.who.int/es/newsroom/detail/30-03-2020-who-releasesguidelines-to-help-countries-maintainessential-health-services-during-thecovid-19-pandemic

16. Gustavo Rodríguez Albor. Cooperación para el desarrollo, relaciones internacionales y políticas públicas. OASIS [Internet]. 2019 Feb 1 [cited 2020 Sep 21];(29). Available from: https://dialnet.unirioja.es/descarga/artic ulo/7016538.pdf

17. Serrano-Cumplido A, Antón-Eguía Ortega PB, Ruiz García A, Olmo Quintana V, Segura Fragoso A, Barquilla Garcia A, et al. COVID-19. History repeats itself and we keep stumbling on the same stone. Semergen. 2020;46 Suppl 1:48-54.

18. Repositorio de datos COVID-19 del Centro de ciencia e ingeniería de sistemas (CSSE) de la Universidad Johns Hopkins [Internet]. github.com. 2020 [citado 24 septiembre 2020]. Disponible en: https://github.com/CSSEGISandData/CO VID-19/

19. Concepción Montiel LE, Suarez $M$, Fernanda Aranibar M. El Impacto De Los
Gobiernos Divididos en La Formulación Y Aprobación De Las Políticas Públicas Legislativas. Nuevo Derecho [Internet]. 2018 Jul [cited 2020 Sep 20];14(23):2-18. Available from: https://dialnet.unirioja.es/servlet/articulo ?codigo $=6742006$

20. Mason D, Gardner D, Hopkins F, O'GradyE. Policy \& Politics in Nursing and Health Care. 7. ${ }^{a}$ ed. St. Louis, Missouri: ELSEVIER; 2020.

21. Gamhewage $G$, Utunen $H$, Attias $M$, George R. Fast-tracking WHO's COVID-19 technical guidance to training for the frontline/Acceleration de la mise a disposition des orientations techniques de I'OMS sur la COVID-19 aux fins de la formation des intervenants de premiere ligne. Weekly Epidemiological Record [Internet]. 2020 [cited 2020 Sep 23];(23):257. Available from: https://apps.who.int/iris/handle/10665/3 32277? locale-attribute $=$ es $\&$

22. Cruz A, Gutiérrez A, Zaldivar E. Gestión de seguridad psicológica del personal sanitario en situaciones de emergencia por COVID-19 en el contexto hospitalario o de aislamiento. Revista Cubana de Enfermería [Internet]. 2020 Apr [cited 2020 Sep 23];36(2):N.PAG. Available from: http://www.revenfermeria.sld.cu/index.p hp/enf/article/view/3704

23. Araceli Damián. Pobreza y desigualdad en México. La construcción ideológica y fáctica de ciudadanías diversas y desiguales*. El Trimestre Económico [Internet]. 2019 Jan 1 [cited 2020 Sep 23];86(343):623-66. Available from: http://www.revistasconacyt.unam.mx/tri mestre/index.php/te/article/view/920/10. 20430/ete.v86i343.920

24. Secretaría de Planeación y Finanzas del Gobierno del Estado de Tlaxcala (SPF). Desarrollo Social [Internet]. SFP.2020 [actualizado septiembre 2020; consultado 
Septiembre 2020]. Available from: https://www.finanzastlax.gob.mx/spf/

25. Constitución Política de los Estados Unidos Méxicanos (UNAM) Ordenamiento-Legislación [Internet]. UNAM.2020 [actualizado mayo 2020; consultado septiembre 2020]. Available from:

https://www.juridicas.unam.mx/legislacio $\mathrm{n} /$ ordenamiento/constitucion-politicade-los-estados-unidosmexicanos\#10560

26. Eguia Casis A. Retos de la auditoría superior de la federación en el año 2018, en el marco del sistema nacional anticorrupción. Revista Ciencia Administrativa [Internet]. 2018 Jul [cited 2020 Sep 23];(2):128-33. Available from: https://cienciasadmvastyp.uat.edu.mx/in dex.php/ACACIA/article/view/255/284

27. Gobierno del Estado de Tlaxcala (TLX), Bienvenida [Internet]. TLX.2020 [actualizado septiembre 2020; consultado septiembre 2020]. Available from: https://www.tlaxcala.gob.mx/index.php/ti tular-sp-24292?id=2663

Copyright (c) 2020 SANUS

Artículo de acceso abierto distribuido bajo Licencia Creative Commons

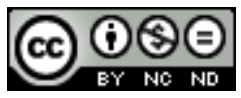

